\title{
NRF2: A potential target for the treatment of diabetic nephropathy
}

Received 23 September 2020

Accepted 3 February 2021

Jiahui Zhang ${ }^{1, *}$, Fnu Anshull ${ }^{1, *}$, Joshua D. Breidenbach ${ }^{1}$, Jing Liu², James Shaffner ${ }^{1}$

'Division of Nephrology, Department of Medicine, University of Toledo College of Medicine, Toledo, OH, USA 2Division of Nephrology, Department of Pediatrics, Vanderbilt University Medical Center, Nashville, TN, USA

\section{Abstract}

One of the major complications of diabetes mellitus is diabetic nephropathy (DN), the pathogenesis of which is primarily driven by oxidative stress. As a major regulator of antioxidant responses, the transcription factor nuclear factor erythroid 2-related factor 2 (NRF2) has recently attracted much interest. NRF2 is a primary defense mechanism against the cytotoxic effects of oxidative stress, involving heterogeneous detoxification, the production of antioxidants and anti-inflammatory molecules, DNA repair, nuclear chaperones, and proteasome systems. A myriad of studies in pre-clinical models of DN have consistently demonstrated a beneficial effect of NRF2 activation, suggesting that NRF2 is likely a promising target for treating DN. This has been further supported by findings from clinical trials of bardoxolone methyl, an activator of NRF2, despite the unexpected adverse cardiovascular effects. This review summarizes the support for therapeutic targeting of NRF2 in DN and emphasizes the need for the optimization of NRF2-based treatment with the minimization of potential adverse effects.

Keywords

diabetic nephropathy $\cdot$ nuclear factor erythroid 2 -related factor $2 \cdot$ oxidative stress

\section{Introduction}

Diabetes mellitus type 1 and type 2 will inevitably result in diabetic nephropathy (DN) at some point, which features a progressive leak of circulating albumin into the urine and thus can be empirically staged according to the severity of the leakage, i.e., normal albuminuria, moderately increased albuminuria (previously known as microalbuminuria), and severely increased albuminuria (previously known as macroalbuminuria) [1-3]. Although long-term epidemiologic studies have shown that not all diabetic patients will progress to DN, it has been estimated that approximately $25-40 \%$ of diabetic patients eventually develop kidney disease [4]. While poor glycemic control is a well-known risk factor for albuminuria and DN [5-7], a large number of patients still develop DN despite adequate glycemic control. Although the complete risk factors for this progression are not fully understood, researchers likely include hereditary, environmental, other pre-existing kidney diseases, and the severity of diabetes [8-10]. Oxidative stress, in particular, is considered to be a key factor for the development of various diabetic complications, including DN [11, 12]. Therefore, as a major regulator of the antioxidant response, nuclear factor erythroid 2-related factor 2 (NRF2) is a potential therapeutic target for the prevention or treatment of $\mathrm{DN}$.

\section{Oxidative Stress: A Key Player in the Pathogenesis of DN}

A consequence of physiological cellular oxygen metabolism is the inevitable production of potentially harmful reactive oxygen species (ROS) [13]. This oxidant formation must be balanced by oxidant removal. Oxidative stress is the result of an imbalance between pro-oxidants and antioxidants which may lead to chronic tissue and organ injury such as DN [11]. Several macromolecules are involved in increased ROS production in the kidney in diabetic conditions [14]. These include $\mathrm{NAD}(\mathrm{P}) \mathrm{H}$ oxidase, advanced glycation end products (AGE), defective polyol pathway, uncoupling of nitric oxide synthase (NOS), and oxidative phosphorylation of mitochondrial respiratory chains [15-18]. Increased abundance of ROS triggers activation of protein kinase $\mathrm{C}$, mitogen-activated protein kinases, and various cytokines and transcription factors. These general inflammatory markers are also common in the pathophysiology of DN and are often found alongside histological lesions including glomerular and tubular basal changes, increased production of extracellular matrix (ECM) which further results in glomerular and tubular basement membrane thickening, dilatation of interstitial matrix of the glomerulus, diffuse glomerulosclerosis, nodular glomerulosclerosis, and hyalinization of afferent arterioles [19-22].

\section{NRF2: The Highly Conserved Master Regulator of Antioxidative Self-defense}

As a result of oxidative stress caused by ROS [23, 24], mammalian cells trigger an effective cytoprotective mechanism in the form of the Cap ' $n$ ' Collar (CNC) basic-leucine zipper (bZip) nuclear transcription factor, NRF2. This defense mechanism includes pathways for xenobiotic detoxification, antioxidant, anti-inflammatory responses, DNA repair, molecular chaperone, and proteasome systems [25, 26].

NRF2, also known as nuclear factor erythroid-derived 2-like 2, is a bZip transcription factor with a CNC structure [27]. 

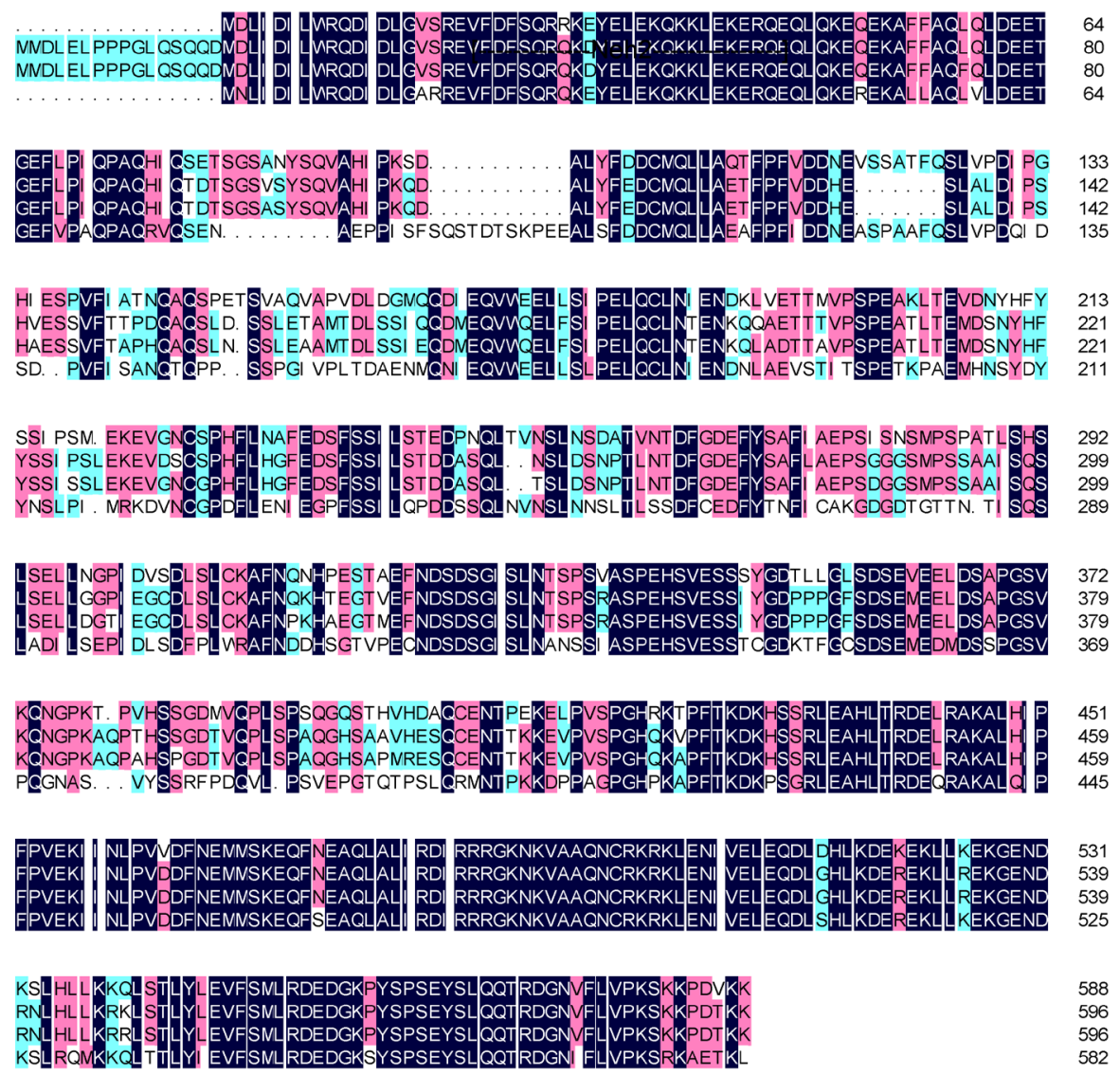

\section{0} (6) 133 142 1 1 11

HUMAN_(HOMO_SAPIENS) RAT_(RATTUS_NORVEGICUS) MOUSE_(MUS_MUSCULUS)

HUMAN_(HOMO_SAPIENS) RAT_(RATTUS_NORVEGICUS) MOUSE_(MUS_MUSCULUS)

HUMAN_(HOMO_SAPIENS) RAT_(RATTUS_NORVEGICUS) CHICKEN_(GA_MUSCULUS)

HUMAN_(HOMO_SAPIENS) RAT_(RATTUS_NORVEGICUS) MOUSE_(MUS MUSCULUS) CHICKEN_(GALLUS_GALLUS)

Figure 1. NRF2, a basic leucine zipper transcription factor. The black box represents completely preserved residues, pink indicates residues with similar properties, blue indicates those with weakly similar properties, and white boxes represent those not identical to human NRF2. NRF2, nuclear factor erythroid 2-related factor 2.

Homologs to human NRF2 exist in various organisms such as rats, mice, and chickens (Figure 1 and Table 1). NRF2 has seven highly conserved domains called NRF2-ECH homology (Neh) domains (Neh1 7 domains). The Neh1 domain, a CNC-bZip domain, is necessary for the formation of a heterodimer with small musculoaponeurotic fibrosarcoma (sMaf) and DNA binding [28]. The Neh2 domain binds with its cytosolic repressor, kelch-like $\mathrm{ECH}$-associated protein 1 (KEAP1), via DLG and ETGE motifs within Neh2, leading to the degradation of NRF2 [29]. The Neh3 domain is necessary for transcriptional activation [30], while Neh4/5 domains are independent transactivation domains [31]. Besides, the recently uncharacterized Neh6 domain is involved in the process of NRF2 degradation [32]. Finally, the Neh7 domain helps to suppress NRF2/antioxidant response element (ARE) via serving as a binding site for $\mathrm{RXR} \alpha$ [33].

\section{Signaling Pathways Regulating the NRF2 Antioxidant Response}

Under basal conditions, NRF2 is restricted to the cytoplasm at very low levels by the binding of the DLG and ETGE motifs of the Neh2 domain to KEAP1, leading to degradation by the proteasome [31]. However, when the cells are exposed to oxidative stress, the binding of the DLG motif of NRF2 to KEAP1 is inhibited, suppressing the rate of degradation [34]. Freed from KEAP1, NRF2 translocates to the nucleus where it forms a heterodimer with sMaf proteins, its obligatory partner, and binds to the ARE sequence initiating the transcription of a multitude of NRF2 downstream genes (Figure 2). These NRF2-regulated genes are classified into intracellular redox-balancing proteins and phase II detoxifying enzymes and transporters, such as glutamate-cysteine ligase (GCL), glutathione s-transferase (GST), and multidrug resistance-associated protein (MRP) [35-37]. As the primary regulator of these cytoprotective genes, the ubiquitously expressed NRF2 is largely responsible for maintaining cellular homeostasis under oxidative stress [38]. Along with the NRF2-mediated protective pathways for toxic or oxidant exposure, NRF2 is also involved in the mechanisms of cellular differentiation, proliferation, inflammation, and lipid synthesis [38, 39].

The activation of NRF2 is under tight regulation, and various diseases including neurodegenerative diseases, malignancy, and cardiovascular diseases are associated with abnormal expression and function of NRF2 [40]. Along with the KEAP1-dependent mechanism of activation described 


\begin{tabular}{llccccc}
\hline Species & Accession & Max score & Total score & Query cover (\%) & E value\% & Identity \\
\hline Homo sapiens & NP_001138884.1 & 1206 & 1206 & $100 \%$ & 0.0 & $100.00 \%$ \\
Rattus norvegicus & NP_113977.1 & 964 & 964 & $100 \%$ & 0.0 & $81.22 \%$ \\
Mus musculus & NP_035032.1 & 952 & 952 & $100 \%$ & 0.0 & $80.54 \%$ \\
Gallus gallus & NP_990448.1 & 726 & 726 & $99 \%$ & 0.0 & $65.82 \%$ \\
\hline
\end{tabular}

Table 1. Protein sequence and homology analysis by NCBI BLAST (https://blast.ncbi.n/m.nih.gov/Blast.cgi) for NRF2 in various organisms

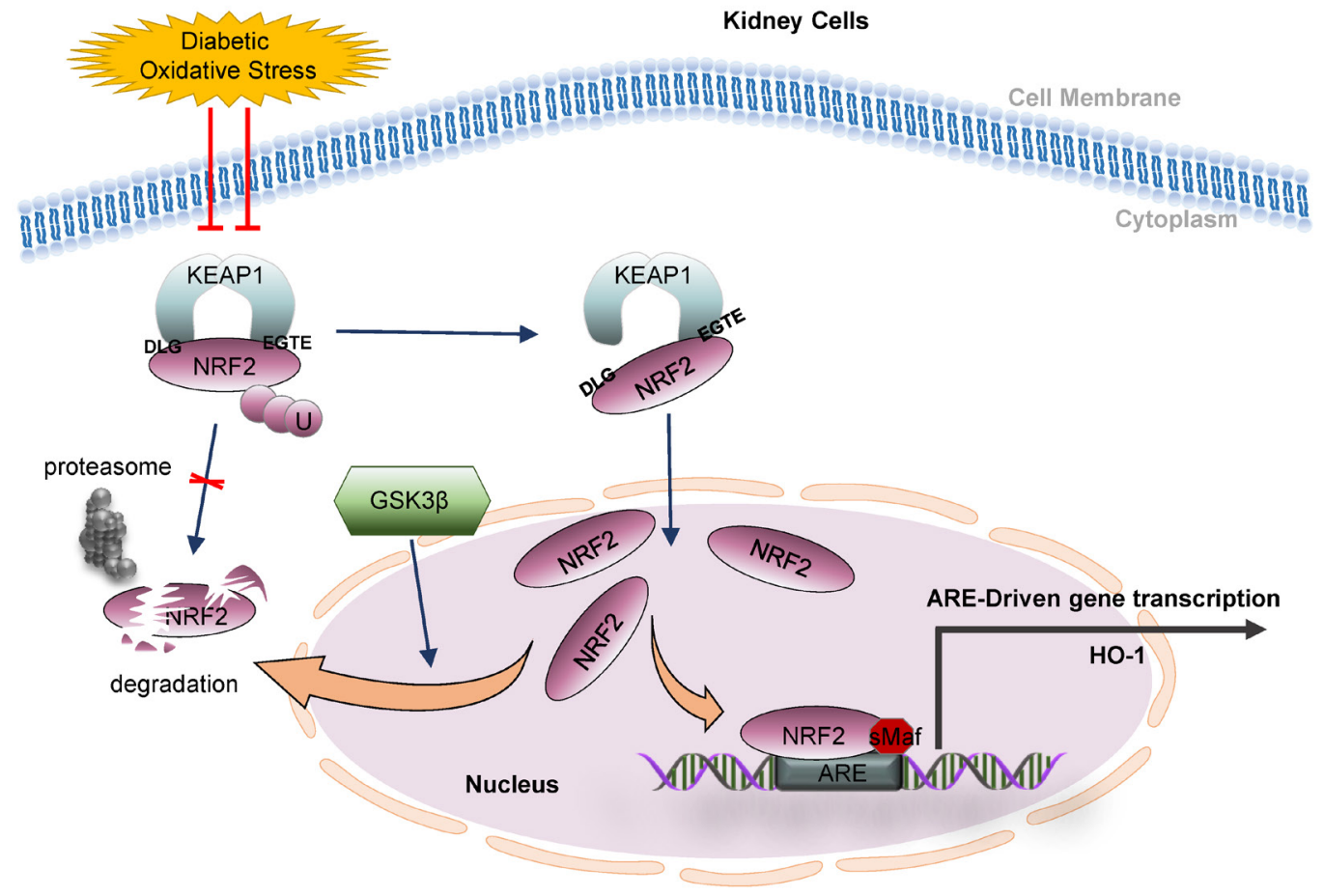

Figure 2. The mechanism diagram illustrates the KEAP1-dependent and GSK3 $\beta$-mediated KEAP1-independent regulation pathway of the NRF2 antioxidant response. Under basal conditions, the DLG and EGTE motifs of NRF2 bind KEAP1 in the cytoplasm. This binding localizes NRF2 to the cytoplasm and causes its ubiquitination and degradation via the proteasome. However, when kidney cells are exposed to diabetic oxidative stress, this degradation is inhibited and NRF2 is separated from KEAP1 at the DLG motif. This structural change causes NRF2 to escape the control of KEAP1 entirely allowing NRF2 nuclear translocation, where NRF2 forms a heterodimer with its essential partner, the sMaf proteins, and binds to the ARE sequence to initiate transcription of several NRF2 downstream genes which eliminate stress or oxidative damage. In response to diabetic oxidative stress, GSK3 $\beta$ is overactivated to promote the excretion of NRF2 from the nucleus and then degradation. ARE, antioxidant response element; GSK3 $\beta$, glycogen synthase kinase 33; HO-1, heme oxygenase 1; KEAP1, kelch-like ECH-associated protein 1; NRF2, nuclear factor erythroid 2-related factor 2; sMaf, small musculoaponeurotic fibrosarcoma.

above, a glycogen synthase kinase $3 \beta$ (GSK3 $\beta$ )-mediated KEAP1-independent mechanism is responsible for NRF2 degradation in the setting of oxidative stress-induced NRF2 $[41,42]$. By the promotion of its nuclear elimination and degradation, GSK3 $\beta$ is a negative regulator of NRF2 (Figure 2). It is important to turn on GSK3 $\beta$ in response to remove NRF2 from the nucleus where it is liberated and migrates into the nucleus, which does not affect the underlying activity of NRF2 but primarily regulates its activity in the later stages of the NRF2 antioxidant response [41]. Several signaling cascades can modulate NRF2 activity through KEAP1-dependent and KEAP1-independent mechanisms, it is important that NRF2 must be expressed correctly, neither too much nor too little, to protect the cell. Therefore, the modulation of targets such as GSK3 $\beta$ may represent additional therapeutic strategies for the KEAP1-independent regulation of NRF2 activity.

\section{Therapeutic Targeting of NRF2 to Treat DN: Evidence from Pre-Clinical Studies}


Nearly all relevant pre-clinical studies have indicated a beneficial effect of NRF2 in DN. Specifically, these studies have shown that the renoprotective activities of several drugs in pre-clinical models of type 1 or $2 \mathrm{DN}$ are mediated through the NRF2-ARE signaling axis. In this regard, the beneficial effects of resveratrol [43], rosuvastatin [44], curcumin [45], salvianolic acid A [46], telmisartan [47], mycophenolate mofetil [48], fenofibrate [49], and hepatocyte growth factor [50] by in vivo or in vitro models of DN have been demonstrated to be conveyed at least in part by activating the NRF2 antioxidant response. Further, these findings were later corroborated by selective silencing of KEAP1, the endogenous NRF2 inhibitor, in kidney cells. More specifically, Zheng et al. [51] demonstrated a reduced expression of transforming growth factor- $\beta$ (TGF $\beta$ ) and matrix proteins in human renal mesangial cells under normal and hyperglycemic conditions by RNA interference of KEAP1. Later, a myriad of studies focused on the development of highly selective small-molecule activators of NRF2, typically triggering indirect activation of NRF2 via the suppression of KEAP1. Tert-butylhydroquinone and sulforaphane, the two most popularly used NRF2 activators, have been shown to alleviate DN in mouse and rat models [52]. With these data, it would be tempting to speculate that KEAP1 knockout animals may be resistant to the development of DN. However, mice with KEAP1 genetic ablation do not survive $>3$ weeks after birth [53], suggesting that targeting KEAP1 chronically to activate NRF2 may yield unintended side effects. In fact, RTA 405, a synthetic triterpenoid analog of bardoxolone methyl, another KEAP1 inhibitor, aggravated proteinuria, glomerulosclerosis, and tubular damage in Zucker diabetic fatty rats with type 2 diabetes (T2D) [54]. These findings suggest that NRF2 antioxidant response is indeed a great therapeutic target for DN but KEAP1 blockade-based NRF2 activation may not be the optimal approach. Further studies are merited to explore other therapeutic strategies to harness the beneficial aspects of NRF2 activation for the treatment or prevention of DN.

\section{Therapeutic Targeting of NRF2 to Treat DN: Evidence from Clinical Trials}

To date, the most extensively studied NRF2 activator in patients with $\mathrm{DN}$ is bardoxolone methyl, also referred to as "RTA 402," "CDDO-methyl ester," and "CDDO-Me." This semisynthetic triterpenoid is based on the scaffold of oleanolic acid and is orally bioavailable [53]. Bardoxolone methyl binds to KEAP1 and inhibits its ubiquitination, which allows for NRF2 nuclear translocation $[55,56]$. The earliest sign of the potential renoprotective activity of bardoxolone was revealed in a retrospective analysis of bardoxolone methyl in advanced solid tumors and lymphomas during a phase I first-in-human trial [57]. In this study, there was an overall $26 \%$ increase in estimated glomerular filtration rate (eGFR) for all patients. Later, in a multinational, randomized, double-blinded, placebo-controlled phase 2 outcome-trial which enrolled 227 adults with T2D and stage IV chronic kidney disease (CKD) (52-week bardoxolone methyl treatment: renal function in CKD/T2D [BEAM] study), demonstrated rapid improvements in the eGFR (20 45 mL/min/1.73 $\left.\mathrm{m}^{2}\right)$. The changes observed within 4 weeks were largely reversible when the drug was stopped due to the direct hemodynamic effect of this strategy [57]. In recent years, typical interventions seem to delay the decline in renal function to $<1 \mathrm{~mL} / \mathrm{min} / 1.73 \mathrm{~m}^{2} /$ year. However, bardoxolone methyl treatment demonstrated improvements of between 5 and $10 \mathrm{~mL} / \mathrm{min} / 1.73 \mathrm{~m}^{2} /$ year. Due to the significantly increased hospitalization and increased mortality resulting from heart failure (96 [8.8\%] with bardoxolone methyl vs. 55 [5\%] to placebo), the BEACON trial was given up [58]. Nevertheless, promising data from bardoxolone trials in other kidney diseases such as Alport Syndrome has rekindled the interest in therapeutic targeting of NRF2 in DN via the use of other NRF2 activators or via optimizing a combined regimen of bardoxolone with cardiovascular drugs [58].

\section{Conclusion}

The pathogenesis of DN is likely to be heavily driven by oxidative stress. As a master regulator of antioxidative self-defense in mammalian cells, NRF2 has attracted much interest in the quest for DN therapy. Given the consistent findings in support of a beneficial effect of activating NRF2 in vitro and in pre-clinical models of DN, it is conceivable that NRF2 is a promising target for treating or preventing DN. Furthermore, clinical trials of bardoxolone, an NRF2 activator, yielded promising results despite unexpected adverse cardiovascular events. Currently, therapeutic targeting of NRF2 in DN is largely limited to KEAP1 inhibitors. Future studies are warranted to explore KEAP1-independent NRF2 regulatory signaling that may be utilized to harness NRF2 for the treatment and possible prevention of DN while minimizing the potential adverse effects.

\section{Author Contributions}

JZ wrote the original draft; JZ and FA contributed to the discussion; JZ, FA, JDB, JL, and JS contributed to the review and editing; and all authors approved the final version of the paper. All authors have read and agreed to the published version of the manuscript. Conflict of Interest

None declared.

\section{Funding Sources}




\section{REFERENCES}

[1] Gross JL, de Azevedo MJ, Silveiro SP, Canani LH, Caramori ML, Zelmanovitz T. Diabetic nephropathy: Diagnosis, prevention, and treatment. Diabetes care 2005; 28:164-76.

[2] Ilyas Z, Chaiban JT, Krikorian A. Novel insights into the pathophysiology and clinical aspects of diabetic nephropathy. Rev Endocr Metab Disord 2017; 18:21-8.

[3] Jones CA, Krolewski AS, Rogus J, Xue JL, Collins A, Warram JH. Epidemic of end-stage renal disease in people with diabetes in the United States population: Do we know the cause? Kidney Int 2005; 67:1684-91.

[4] Alicic RZ, Rooney MT, Tuttle KR. Diabetic kidney disease: Challenges, progress, and possibilities. Clin J Am Soc Nephrol 2017; 12:2032-45.

[5] Papadopoulou-Marketou N, Paschou SA, Marketos N, Adamidi S, Adamidis S, Kanaka-Gantenbein C. Diabetic nephropathy in type 1 diabetes. Minerva Med 2018; 109: 218-28.

[6] Fullerton B, Jeitler K, Seitz M, Horvath K, Berghold A, Siebenhofer A. Intensive glucose control versus conventional glucose control for type 1 diabetes mellitus. Cochrane Database Syst Rev 2014; 2014:CD009122.

[7] Diabetes Control and Complications Trial Research Group; Nathan DM, Genuth S, Lachin J, Cleary P, Crofford O, et al. The effect of intensive treatment of diabetes on the development and progression of long-term complications in insulin-dependent diabetes mellitus. N Engl J Med 1993; 329:977-86.

[8] Macisaac RJ, Ekinci El,Jerums G. Markers of and risk factors for the development and progression of diabetic kidney disease. Am J Kidney Dis 2014; 63:S39-62.

[9] Regele F, Jelencsics K, Shiffman D, Paré G, McQueen MJ, Mann JFE, et al. Genome-wide studies to identify risk factors for kidney disease with a focus on patients with diabetes. Nephrol Dial Transplant 2015; 30 Suppl 4:iv26-34.

[10] Wang G, Ouyang J, Li S, Wang H, Lian B, Liu Z, et al. The analysis of risk factors for diabetic nephropathy progression and the construction of a prognostic database for chronic kidney diseases. $J$ Transl Med 2019; 17:264.

[11] Jha JC, Banal C, Chow BS, Cooper ME, Jandeleit-Dahm K. Diabetes and kidney disease: Role of oxidative stress. Antioxid Redox Signal 2016; 25:657-84.

[12] Sagoo MK, Gnudi L. Diabetic nephropathy: Is there a role for oxidative stress? Free Radic Biol Med 2018; 116: $50-63$.

[13] Pizzino G, Irrera N, Cucinotta M, Pallio G, Mannino F, Arcoraci $V$, et al. Oxidative stress: Harms and benefits for human health. Oxid Med Cell Longev 2017; 2017:8416763.

[14] Kashihara N, Haruna Y, Kondeti VK, Kanwar YS. Oxidative stress in diabetic nephropathy. Curr Med Chem 2010; 17:4256-69.

[15] Inoguchi T, Sonta T, Tsubouchi H, Etoh T, Kakimoto M, Sonoda $\mathrm{N}$, et al. Protein kinase C-dependent increase in reactive oxygen species (ROS) production in vascular tissues of diabetes: Role of vascular NAD(P)H oxidase. J Am Soc Nephrol 2003; 14:S227-32.

[16] Vlassara H, Palace MR. Diabetes and advanced glycation endproducts. J Intern Med 2002; 251:87-101.

[17] Araki E, Nishikawa T. Oxidative stress: A cause and therapeutic target of diabetic complications. J Diabetes Investig 2010; 1:90-6.
[18] Joseph D, Kimar C, Symington B, Milne R, Essop MF. The detrimental effects of acute hyperglycemia on myocardial glucose uptake. Life Sci 2014; 105:31-42.

[19] Xiang H, Xue W, Wu X, Zheng J, Ding C, Li Y, et al. FOXP1 inhibits high glucose-induced ECM accumulation and oxidative stress in mesangial cells. Chem Biol Interact 2019; 313:108818.

[20] Sifuentes-Franco S, Padilla-Tejeda DE, Carrillo-Ibarra S, MirandaDíaz AG. Oxidative stress, apoptosis, and mitochondrial function in diabetic nephropathy. Int J Endocrinol 2018; 2018:1875870.

[21] Mi X, Tang W, Chen X, Liu F, Tang X. Mitofusin 2 attenuates the histone acetylation at collagen IV promoter in diabetic nephropathy. J Mol Endocrinol 2016; 57:233-49.

[22] Ruiz S, Pergola PE, Zager RA, Vaziri ND. Targeting the transcription factor Nrf2 to ameliorate oxidative stress and inflammation in chronic kidney disease. Kidney Int 2013; 83:1029-41.

[23] Zhang J, Wang X, Vikash V, Ye Q, Wu D, Liu Y, et al. ROS and ROS-mediated cellular signaling. Oxid Med Cell Longev 2016; 2016:4350965

[24] Ray PD, Huang BW, Tsuji Y. Reactive oxygen species (ROS) homeostasis and redox regulation in cellular signaling. Cell Signal 2012; 24:981-90.

[25] Osburn WO, Kensler TW. Nrf2 signaling: An adaptive response pathway for protection against environmental toxic insults. Mutat Res 2008; 659:31-9.

[26] Tu W, Wang H, Li S, Liu Q, Sha H. The anti-inflammatory and anti-oxidant mechanisms of the Keap1/Nrf2/ARE signaling pathway in chronic diseases. Aging Dis 2019; 10: 637-51.

[27] Moi P, Chan K, Asunis I, Cao A, Kan YW. Isolation of NF-E2related factor 2 (Nrf2), a NF-E2-like basic leucine zipper transcriptional activator that binds to the tandem NF-E2/AP1 repeat of the beta-globin locus control region. Proc Natl Acad Sci U S A 1994; 91:9926-30.

[28] Sun Z, Chin YE, Zhang DD. Acetylation of Nrf2 by p300/CBP augments promoter-specific DNA binding of Nrf2 during the antioxidant response. Mol Cell Biol 2009; 29:2658-72.

[29] Tong KI, Kobayashi A, Katsuoka F, Yamamoto M. Twosite substrate recognition model for the Keap1-Nrf2 system: A hinge and latch mechanism. Biol Chem 2006; 387: 1311-20.

[30] Nioi P, Nguyen T, Sherratt PJ, Pickett CB. The carboxy-terminal Neh3 domain of Nrf2 is required for transcriptional activation. Mol Cell Biol 2005; 25:10895-906.

[31] Motohashi H, Yamamoto M. Nrf2-Keap1 defines a physiologically important stress response mechanism. Trends Mol Med 2004; 10:549-57.

[32] McMahon M, Thomas N, Itoh K, Yamamoto M, Hayes JD. Redoxregulated turnover of Nrf2 is determined by at least two separate protein domains, the redox-sensitive Neh2 degron and the redox-insensitive Neh6 degron. J Biol Chem 2004; 279:31556-67.

[33] Nam LB, Keum YS. Binding partners of NRF2: Functions and regulatory mechanisms. Arch Biochem Biophys 2019; 678:108184.

[34] Kaspar JW, Niture SK, Jaiswal AK. Nrf2:INrf2 (Keap1) signaling in oxidative stress. Free Radic Biol Med 2009; 47:1304-9.

[35] Zhang DD. Mechanistic studies of the Nrf2-Keap1 signaling pathway. Drug Metab Rev 2006; 38:769-89. 
[36] Ishii T, Itoh K, Takahashi S, Sato H, Yanagawa T, Katoh $\mathrm{Y}$, et al. Transcription factor Nrf2 coordinately regulates a group of oxidative stress-inducible genes in macrophages. J Biol Chem 2000; 275:16023-9.

[37] Lau A, Villeneuve NF, Sun Z, Wong PK, Zhang DD. Dual roles of Nrf2 in cancer. Pharmacol Res 2008; 58:262-70.

[38] Bryan HK, Olayanju A, Goldring CE, Park BK. The Nrf2 cell defence pathway: Keap1-dependent and - independent mechanisms of regulation. Biochem Pharmacol 2013; 85:705-17.

[39] Murakami S, Motohashi H. Roles of Nrf2 in cell proliferation and differentiation. Free Radic Biol Med 2015; 88:168-78.

[40] Sivandzade F, Bhalerao A, Cucullo L. Cerebrovascular and neurological disorders: Protective role of NRF2. Int J Mol Sci 2019; 20:3433 [41] Lu M, Wang P, Qiao Y, Jiang C, Ge Y, Flickinger B, et al. GSK3ßmediated Keap1-independent regulation of Nrf2 antioxidant response: A molecular rheostat of acute kidney injury to chronic kidney disease transition. Redox Biol 2019; 26:101275.

[42] Zhang J, Anshul F, Malhotra DK, Jaume J, Dworkin LD, Gong R. Microdose lithium protects against pancreatic islet destruction and renal impairment in streptozotocin-elicited diabetes. Antioxidants (Basel) 2021; 10:138

[43] Turan B, Tuncay E, Vassort G. Resveratrol and diabetic cardiac function: Focus on recent in vitro and in vivo studies. J Bioenerg Biomembr 2012; 44:281-96.

[44] Hussein MM, Mahfouz MK. Effect of resveratrol and rosuvastatin on experimental diabetic nephropathy in rats. Biomed Pharmacother 2016; 82:685-92.

[45] Trujillo J, Chirino YI, Molina-Jijón E, Andérica-Romero AC, Tapia E, Pedraza-Chaverrí J. Renoprotective effect of the antioxidant curcumin: Recent findings. Redox Biol 2013; 1:448-56.

[46] Zhang H-F, Wang J-H, Wang Y-L, Gao C, Gu Y-T, Huang J, et al. Salvianolic acid $A$ protects the kidney against oxidative stress by activating the Akt/GSK-3 $\beta /$ Nrf2 signaling pathway and inhibiting the NF-KB signaling pathway in $5 / 6$ nephrectomized rats. Oxid Med Cell Longev 2019; 2019:2853534.

[47] Guo Z, Yan X, Wu J, Jing X, Liu J. Effect of telmisartan or insulin on the expression of adiponectin and its receptors in the testis of streptozotocin-induced diabetic rats. Horm Metab Res 2016; 48:404-12.
[48] Arellano-Buendía AS, Tostado-González M, García-Arroyo FE, Cristóbal-García M, Loredo-Mendoza ML, Tapia E, et al. Antiinflammatory therapy modulates Nrf2-Keap1 in kidney from rats with diabetes. Oxid Med Cell Longev 2016; 2016:4693801.

[49] Cheng Y, Zhang J, Guo W, Li F, Sun W, Chen J, et al. Upregulation of Nrf2 is involved in FGF21-mediated fenofibrate protection against type 1 diabetic nephropathy. Free Radic Biol Med 2016; 93:94-109.

[50] Guoguo S, Akaike T, Tao J, Qi C, Nong Z, Hui L. HGF-mediated inhibition of oxidative stress by 8-nitro-cGMP in high glucose-treated rat mesangial cells. Free Radic Res 2012; 46:1238-48.

[51] Zheng H, Whitman SA, Wu W, Wondrak GT, Wong PK, Fang $D$, et al. Therapeutic potential of Nrf2 activators in streptozotocin-induced diabetic nephropathy. Diabetes 2011; 60:3055-66.

[52] Lazaro I, Lopez-Sanz L, Bernal S, Oguiza A, Recio C, Melgar A, et al. Nrf2 activation provides atheroprotection in diabetic mice through concerted upregulation of antioxidant, anti-inflammatory, and autophagy mechanisms. Front Pharmacol 2018; 9:819.

[53] Wakabayashi N, Itoh K, Wakabayashi J, Motohashi H, Noda S, Takahashi S, et al. Keap1-null mutation leads to postnatal lethality due to constitutive Nrf2 activation. Nat Genet 2003; 35:238-45.

[54] Zoja C, Corna D, Nava V, Locatelli M, Abbate M, Gaspari F, et al. Analogs of bardoxolone methyl worsen diabetic nephropathy in rats with additional adverse effects. Am J Physiol Renal Physiol 2013; 304:F808-19.

[55] Yates MS, Tauchi M, Katsuoka F, Flanders KC, Liby KT, Honda $\mathrm{T}$, et al. Pharmacodynamic characterization of chemopreventive triterpenoids as exceptionally potent inducers of Nrf2-regulated genes. Mol Cancer Ther 2007; 6:154-62.

[56] Dinkova-Kostova AT, Liby KT, Stephenson KK, Holtzclaw WD, Gao X, Suh N, et al. Extremely potent triterpenoid inducers of the phase 2 response: Correlations of protection against oxidant and inflammatory stress. Proc Natl Acad Sci U S A 2005; 102:4584-9.

[57] Hong DS, Kurzrock R, Supko JG, He X, Naing A, Wheler J, et al. A phase I first-in-human trial of bardoxolone methyl in patients with advanced solid tumors and lymphomas. Clin Cancer Res 2012; 18:3396-406.

[58] Toto RD. Bardoxolone-the Phoenix? J Am Soc Nephrol 2018; 29:360-1. 